\title{
Effect of element type on the internal bond quality of wood-based panels determined by three methods
}

\begin{abstract}
Three mechanical tests with different loading modes were conducted to evaluate the effect of element type on the internal bond quality of wood-based panels. In addition to the internal bond test, which is commonly used for mat-formed panels, interlaminar and edgewise shear tests were used to test oriented strandboard (OSB), particleboard, medium-density fiberboard (MDF) of two thicknesses, and plywood. The following results were obtained. Epoxy resin proved to be suitable for determining the interlaminar shear modulus instead of hot-melt glue. There was a linear relation between panel density and interlaminar shear modulus and a linear correlation between the interlaminar shear strength and internal bond (IB) strength for the mat-formed panels tested. OSB had the highest edgewise shear modulus, and MDFs had the highest edgewise shear strength in this study. The modulus/strength ratio also depended on both panel type and loading mode. The relation between the shear moduli determined from the edgewise and interlaminar tests indicated the characteristics of the shear properties of panels made of different elements.
\end{abstract}

Key words Internal bond strength - Interlaminar shear property $\cdot$ Edgewise shear property $\cdot$ Wood-based panels

\section{Introduction}

The annual supply of mat-formed panels, such as oriented strandboard (OSB), particleboard (PB), and medium-

S. Suzuki $(\bowtie) \cdot H$. Miyagawa

Faculty of Agriculture, Shizuoka University, Shizuoka 422-8529,

Japan

Tel. +81-54-238-4854; Fax +81-54-237-3028

e-mail: afssuzu@ipc.shizuoka.ac.jp

Part of this paper was presented at the Fourth International Wood Science Symposium, Serpong, Indonesia, September 2002 density fiberboard (MDF), has been increasing because of shortages in plywood production. Because these products have a large number of internal wood-resin interfaces, their physical and mechanical properties are greatly influenced by the quality of bonding between elements, called internal bonds. Internal bond quality also depends on the depth between the surfaces of the panel, as a vertical density gradient forms during pressing and consolidation of the mat. The tensile strength perpendicular to the surface (IB strength) is commonly used to determine the internal bond quality; this is regarded, however, as representing only the strength of the weakest layer in the panel.

This paper discusses the internal bond quality of matformed panel products by focusing on three loading modes, as shown in Fig. 1. IB strength, obtained by loading in direction (1), is used as a quality control measure in plants, and the test method is specified in standards such as the ASTM $,{ }^{1} \mathrm{EN}^{2}{ }^{2} \mathrm{JIS},{ }^{3}$ and $\mathrm{JAS} .{ }^{4}$ IB strength is considered the most important mechanical property of panel products, not only for industrial use but also for research and laboratoryscale tests. The mechanical properties quantified by loading mode (2) also reflect the internal bond quality of a panel. The shear test in which a force is applied along the plane of the panel is called the interlaminar shear test. It is specified in $\mathrm{ASTM}^{1}$ and prEN. ${ }^{5}$ Other methods, such as the block shear test and the Minnesota shear test, ${ }^{1,6,7}$ quantify the interlaminar shear properties. The shear-through-thickness property obtained by loading mode (3) is another measure that is influenced by the bond quality between elements in the lateral direction in a panel. The ASTM D 1037 specifies the test procedure for the edgewise shear test ${ }^{1}$ of woodbased panel products.

The IB strength and interlaminar shear strength are thought to be closely related because failures in both tests occur in the core layer, and both strengths are indications of the bond quality between elements in the core layer of matformed panels. Some studies have examined this correlation. McNatt ${ }^{8}$ reported a high correlation between the shear and IB strengths of particleboards, and Schulte and Fruewald ${ }^{9}$ found a correlation for MDFs according to prEN 789. ${ }^{5}$ Suzuki et al. ${ }^{10}$ compared the IB strength of MDF with 
the interlaminar shear strength obtained by a short-beam bending test. Research dealing with interlaminar shear has sought alternatives to the IB test. The validity of the fivepoint bending test, ${ }^{11}$ a new compression shear test, ${ }^{12}$ and a block shear test ${ }^{13}$ method has been evaluated. Torsion tests $^{14-17}$ have also been proposed as a method for determining shear strength. Despite these efforts, we have little information on interlaminar shear properties because no test methods for determining them are specified in the current standards in Japan.

Research on shear properties in the through-thethickness direction (edgewise shear) is limited compared to that on interlaminar shear. For example, $\mathrm{McNatt}^{8}$ reported the edgewise shear strength of several particle panel products, and Suzuki et al. ${ }^{18}$ evaluated the shear-through-thickness of wood-based panels using the two-rail shear and edgewise shear test methods. Lee and Stephens ${ }^{19}$ obtained the edgewise and interlaminar shear strengths for seven commercial wood-based panel products. The relation between the interlaminar and edgewise shear is one concern when discussing the effect of the element type on the internal bond quality of wood-based panels.

The objectives of this study were to evaluate the effects of the element type on the bond quality inside woodbased panels using three mechanical tests with different loading modes - IB test, interlaminar shear test, edgewise shear test - and to determine the relations between these properties.

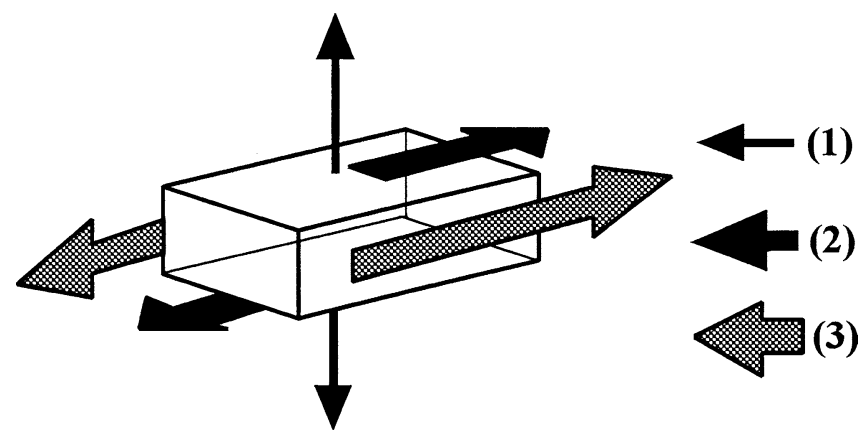

Fig. 1. Loading modes used to evaluate the internal bond quality of mat-formed panels

\section{Materials and methods}

Wood-based panels used

The five commercial wood-based panels examined in this study are described in Table 1. OSB and plywood (PW) were JAS structural grade, and PB was JIS high moistureresistant grade. MDFs of two nominal thicknesses were both classified as M-type in the JIS. Ten test sheets of each panel type were obtained. The interlaminar and edgewise shear test specimens were prepared to test these panels in both the parallel $(\mathrm{Pr})$ and perpendicular $(\mathrm{Pp})$ directions. The direction was defined as the machine direction for $\mathrm{PB}$ and MDF and as the face fiber grain alignment for OSB and PW.

Internal bond test

The internal bond (IB) strength was determined according to JIS $5908 .^{3}$ There were 10 replicates for each panel type.

\section{Interlaminar shear test}

Testing was performed according to ASTM D $1037^{1}$ using 16 specimens of MDF and 13 specimens of OSB, PB, and PW for each direction. Steel plates were bonded to each face of the test specimen using epoxy resin adhesive or hotmelt glue. The specimens were clamped for about $24 \mathrm{~h}$ to ensure a good glue bond. A load was applied to the steel plates at a rate of $2 \mathrm{kN} / \mathrm{min}$. Displacement of one plate with respect to the other was measured using a dial gauge-type displacement transducer. The interlaminar shear strength (TILS) and modulus of rigidity (GILS) were calculated using the equations.

$$
\begin{aligned}
& \text { TILS }=P_{\max } /(L \cdot b) \\
& \text { GILS }=P \cdot d /(L \cdot b \cdot r)
\end{aligned}
$$

where $b, d$, and $L$ are the width, thickness, and length of the specimen in millimeters, respectively; $P_{\max }$ is the maximum load (newtons) at failure; $P$ is the increment of the load on the straight-line portion of the load-displacement curve; and $r$ is the increment of displacement corresponding to $P$. GILS was calculated from the portion between $10 \%$ and

\begin{tabular}{|c|c|c|c|c|}
\hline Panels & Symbol & $\begin{array}{l}\text { Thickness } \\
(\mathrm{mm})\end{array}$ & $\begin{array}{l}\text { Density } \\
\left(\mathrm{g} / \mathrm{cm}^{3}\right)\end{array}$ & Remarks \\
\hline Oriented strandboard & OSB & 10.1 & 0.65 & $\begin{array}{l}\text { JAS structural panel, class } 4 \text {, } \\
\text { three-layer }\end{array}$ \\
\hline Particleboard & $\mathrm{PB}$ & 9.2 & 0.78 & JIS A 5908, P-18 type \\
\hline \multirow[t]{2}{*}{$\begin{array}{l}\text { Medium-density } \\
\text { fiberboard }\end{array}$} & MDF9 & 9.1 & 0.74 & JIS A 5905, M-30 type \\
\hline & MDF7 & 7.2 & 0.76 & JIS A 5905, M-30 type \\
\hline Plywood & PW & 8.8 & 0.57 & $\begin{array}{l}\text { JAS structural plywood, class } 2 \text {, } \\
\text { three-ply }\end{array}$ \\
\hline
\end{tabular}

Table 1. Type of panels tested 
$40 \%$ of the maximum load. Additionally, the shear deformation of the gluelines was measured using a steel specimen. A 4-mm-thick steel specimen was bonded between the loading plates, instead of a wooden specimen, using each adhesive.

\section{Edgewise shear test}

Testing was performed according to ASTM D 1037. ${ }^{1}$ Four steel rails with a serrated gripping surface were bolted to a $254 \times 90 \mathrm{~mm}$ test specimen. The rails were bolted tightly to prevent slipping and loaded at a rate of $2 \mathrm{kN} / \mathrm{min}$ in compression to produce shear deformation. The strain on the specimen was measured using two wire strain gauges placed at 45 degrees to the center line, one on each face, in the middle of the specimen. The edgewise shear strength (TEWS) and modulus of rigidity (GEWS) were calculated using the equations

$$
\begin{aligned}
& \text { TEWS }=P_{\max } /(L \cdot t) \\
& \mathrm{GEWS}=P /(2 \cdot L \cdot t \cdot e)
\end{aligned}
$$

where $t$ is the thickness (millimeters), $e$ is the shear strain of the specimen, and $P_{\max }$ is the maximum load (newtons) at failure. GEWS was calculated from the straight-line portion between $5 \%$ and $40 \%$ of maximum load on the loaddeformation curve.

\section{Results and discussion}

Internal bond strength

Internal bond strength is one of the most important indications of the internal bond quality of mat-formed panels, and the IB test is widely used. The mean IB strengths and coefficients of variation (CV) are given in Table 2. PW had the highest IB strength but also had greater variability than the other panels. The adhesive strength of plywood is generally evaluated by the tensile shear test between veneers, and no IB test method for this is specified in the standards. The IB strength of PW was obtained to compare with that of matformed panels. MDF7 had the highest IB strength among the mat-formed panels, and OSB and MDF9 had the lowest values. The coefficient of variation of OSB was larger than that of PB and MDF owing to the relatively large element size in this type of panel.

Table 2. Internal bond strength of the panels

\begin{tabular}{llc}
\hline Panel type & IB $(\mathrm{MPa})$ & CV $(\%)$ \\
\hline OSB & 0.59 & 18 \\
PB & 1.07 & 8 \\
MDF9 & 0.57 & 11 \\
MDF7 & 1.21 & 16 \\
PW & 1.27 & 28
\end{tabular}

IB, internal bond strength; $\mathrm{CV}$, coefficient of variation
Interlaminar shear

\section{Effect of resin type on displacement}

The interlaminar shear (ILS) test is not considered complicated when the test is conducted only to obtain the fracture load for the shear strength. Hot-melt glue can be used for this purpose to shorten the testing period. Some researchers ${ }^{19,20}$ have determined the shear strength of panel products using this method. Few studies ${ }^{8,9,11}$ have determined both the strength and the shear modulus with the ILS test. It is time-consuming and laborious to obtain the shear modulus because the ILS test piece must be rigidly glued between the plates and the displacement transducer must be attached to an exact position on the specimen.

To evaluate the effect of resin type for better gluing, an ILS test using a steel piece was performed. Figure 2 shows the relation between load and displacement when a 4-mmthick steel specimen was bonded between the loading plates using two different glues. Displacement of the two loading plates of about 0.03 and $0.06 \mathrm{~mm}$ at loads of 15 and $20 \mathrm{kN}$,

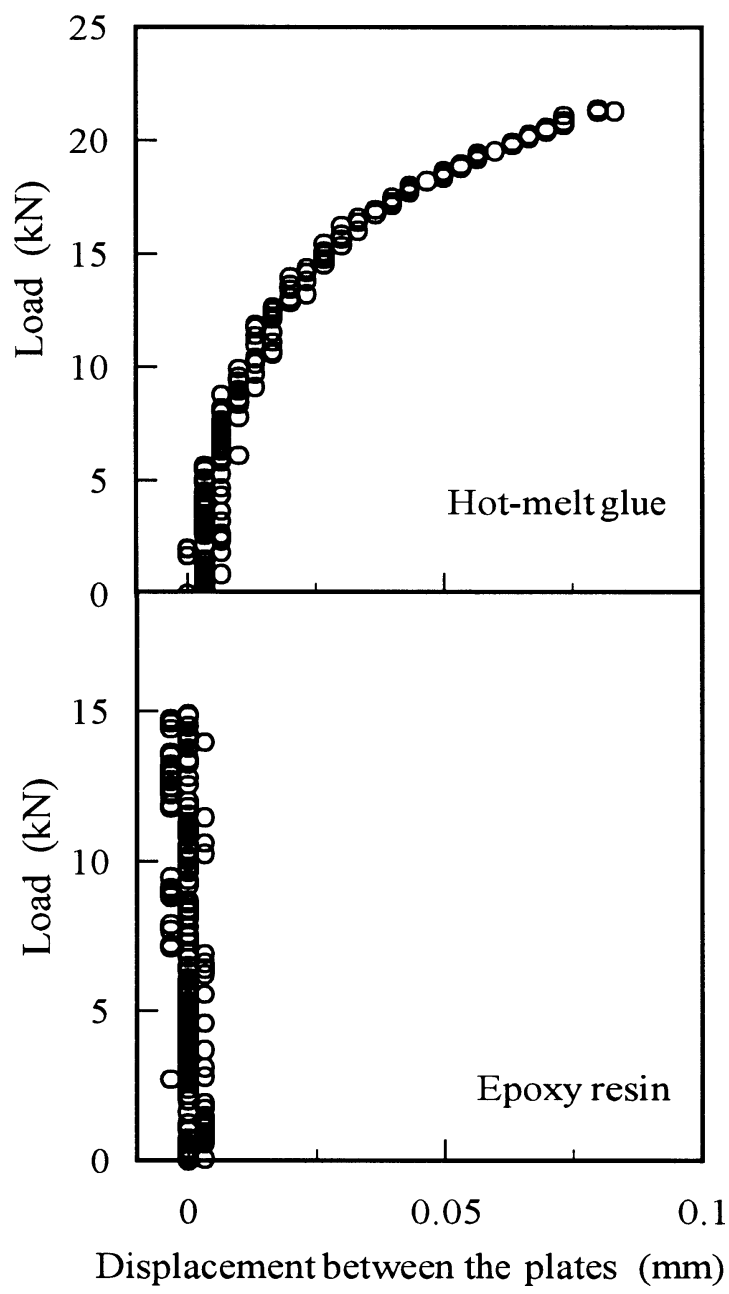

Fig. 2. Relation between load and displacement in the interlaminar shear (ILS) test using various glues to bond the steel specimen between the loading plates. Top Bonded with hot-melt glue. Bottom Bonded with epoxy resin 


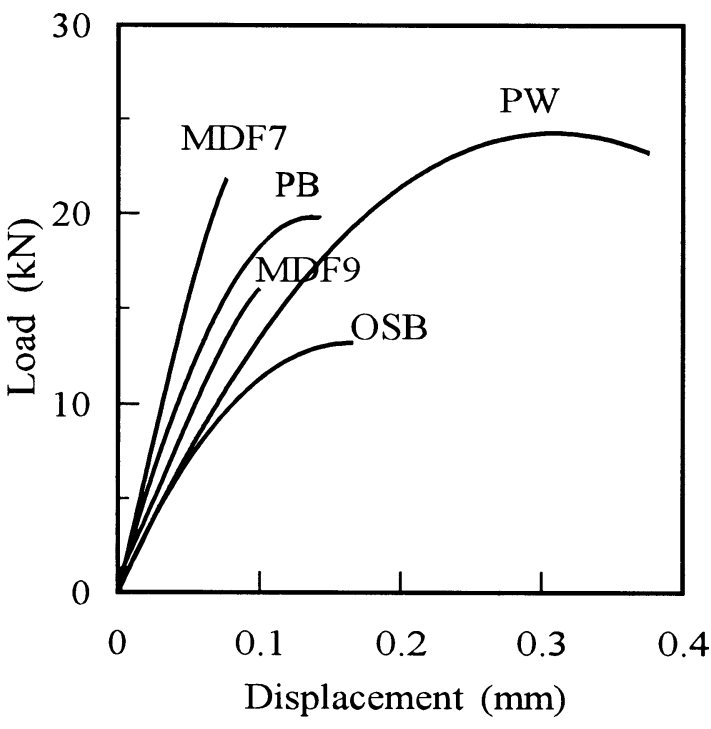

Fig. 3. Relation between load and displacement between the plates in the ILS test. $M D F$, medium-density fiberboard; $P B$, particleboard; $P W$, plywood; $O S B$, oriented stransboard

respectively, was observed with hot-melt glue. When using hot-melt glue for the ILS test, the calculated modulus might be somewhat lower than the true value, as the deformation could include slip in the glueline. The hot-melt glue proved to have insufficient bonding strength for estimating the shear modulus. By contrast, no slip between the plates was observed for epoxy resin, as shown in Fig. 2. Although both resins provided a bond sufficient for determining the shear strength, these results showed that epoxy resin is suitable for GILS measurement. When using epoxy resin, the loading plates must be sanded and cleaned to provide a good bond and prevent failure along the glueline.

\section{Shear properties determined with the ILS test}

Some typical load-deformation curves of parallel specimens bonded to the plates with epoxy resin are shown in Fig. 3. For all panel types, the shear modulus (GILS) could be obtained from a linear regression of the loaddeformation data. This linear relation held for a portion of the range. The MDFs failed rapidly, and the PB and OSB failed somewhat rapidly after reaching the limit of this range. By contrast, PW failed with an exponential increase in deformation after reaching the limit. This showed that the deformation depended on the panel elements, and that the mat-formed panel products were more brittle than PW when subjected to interlaminar shear loading. This ductile property of $\mathrm{PW}$, compared to the other materials, is one of the typical characteristics of veneer-based panels.

The mean interlaminar shear strengths and moduli of the five panel types are given in Table 3. The GILS was approximately $290 \mathrm{MPa}$ for PB and $200 \mathrm{MPa}$ for OSB. These values are comparable to the results for commercial PB and OSB reported by $\mathrm{McNatt}{ }^{8}$ and Bateman et al., ${ }^{11}$ respectively. The GILS was higher for MDF and PB than for OSB and PW. There is little information on this shear modulus for differ-

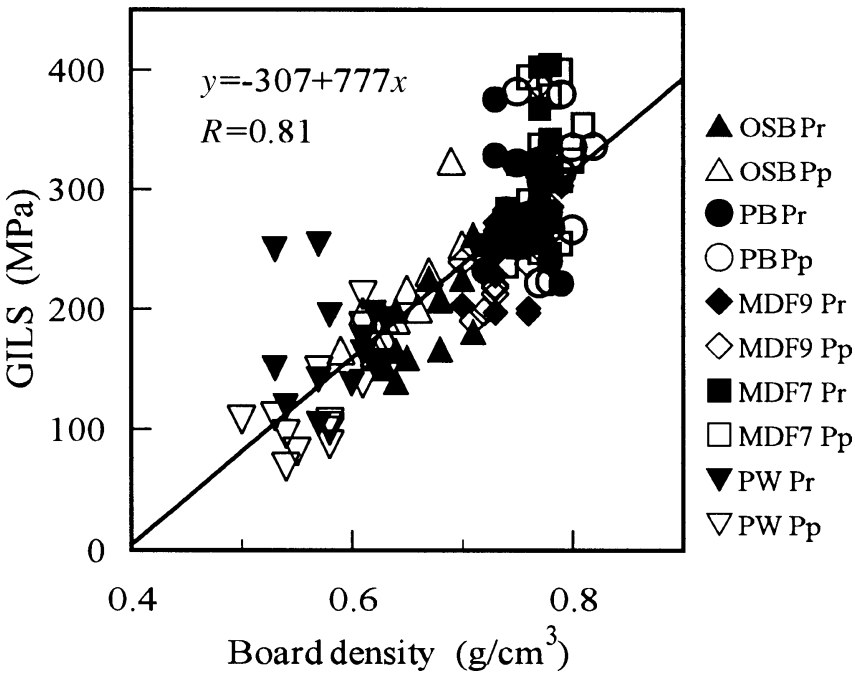

Fig. 4. Relation between board density and shear modulus in the ILS test. $P r$, parallel to the machine direction or face fiber grain alignment; $P p$, perpendicular to the machine direction or the face fiber grain alignment. GILS, interlaminar shear modulus

Table 3. Shear properties obtained by the interlaminar shear test

\begin{tabular}{|c|c|c|c|c|c|}
\hline \multirow[t]{2}{*}{ Panel type } & \multicolumn{2}{|c|}{ GILS } & \multicolumn{2}{|l|}{ TILS } & \multirow[t]{2}{*}{ GILS/TILS } \\
\hline & $\mathrm{MPa}$ & CV (\%) & $\mathrm{MPa}$ & CV (\%) & \\
\hline \multicolumn{6}{|l|}{ OSB } \\
\hline $\operatorname{Pr}$ & 188 & 19 & $1.74 *$ & 8 & 108 \\
\hline Pp & 205 & 21 & $2.16^{*}$ & 12 & 95 \\
\hline \multicolumn{6}{|l|}{$\mathrm{PB}$} \\
\hline $\operatorname{Pr}$ & 291 & 15 & 2.66 & 6 & 109 \\
\hline $\mathrm{Pp}$ & 300 & 17 & 2.78 & 5 & 108 \\
\hline \multicolumn{6}{|l|}{ MDF9 } \\
\hline $\operatorname{Pr}$ & 248 & 14 & 2.15 & 10 & 115 \\
\hline $\mathrm{Pp}$ & 257 & 19 & 2.11 & 11 & 121 \\
\hline \multicolumn{6}{|l|}{ MDF7 } \\
\hline $\operatorname{Pr}$ & 300 & 17 & 3.03 & 7 & 99 \\
\hline $\mathrm{Pp}$ & 309 & 17 & 3.11 & 9 & 99 \\
\hline \multicolumn{6}{|l|}{ PW } \\
\hline $\operatorname{Pr}$ & $171^{*}$ & 25 & $3.07 *$ & 19 & 56 \\
\hline $\mathrm{Pp}$ & $118^{*}$ & 34 & $2.04 *$ & 18 & 58 \\
\hline
\end{tabular}

GILS, interlaminar shear modulus; TILS, interlaminar shear strength; Pr, parallel to the machine direction or face fiber grain alignment; $\mathrm{Pp}$, perpendicular to the machine direction or the face fiber grain alignment

* Significantly different at the $5 \%$ level

ent panel types; indeed, this is the first paper to discuss the effect of panel density in this respect. Figure 4 shows the relation between density and GILS. There was a linear relation despite the differences in elements, face alignments, and resin types among the panels. The following regression equation was obtained

$y=-307+777 x \quad R=0.81$

where $y$ is the GILS (MPa), and $x$ is the panel density (g/ $\mathrm{cm}^{3}$ ). We concluded that the density affects the interlaminar shear modulus as it affects the other mechanical properties. The density profile in the thickness direction of the panels 


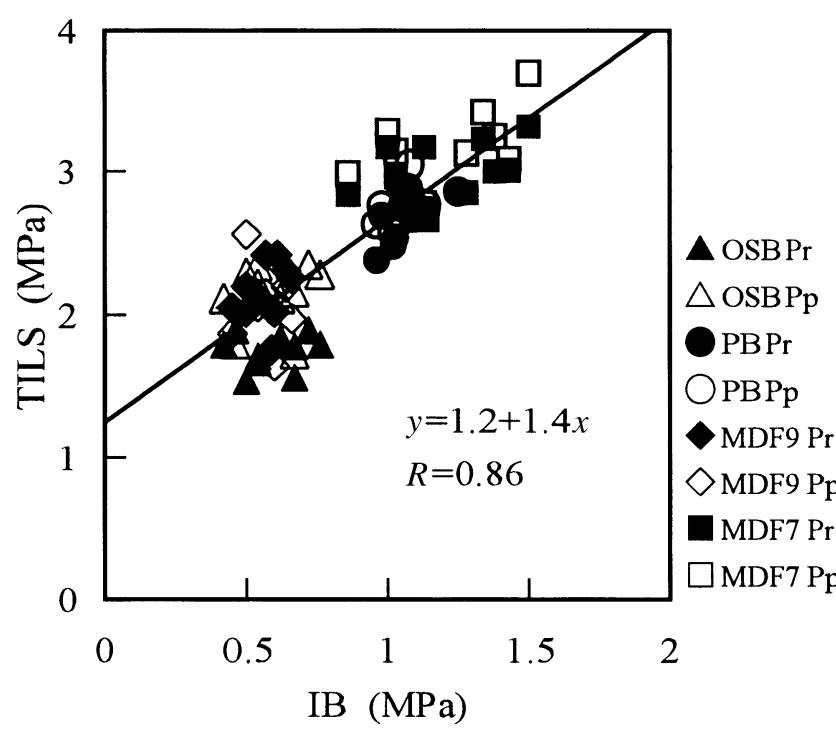

Fig. 5. Relation between internal bond $(I B)$ strength and interlaminar shear strength (TILS)

will be one of the viewpoints when discussing interlaminar shear properties in future work.

The shear strength (TILS) of each panel is also shown in Table 3. PW had the lowest GILS but the highest TILS in the parallel direction. Comparing the TILS of mat-formed panels, the trend was similar to that for GILS; the mean value decreased in the order MDF7, PB, MDF9, and OSB. Because there was a marked difference between the testing directions in the TILS of PW, the $t$-test was used to evaluate the effects of machine direction and face grain alignment on the interlaminar shear properties. No statistical differences were found for $\mathrm{PB}$ or MDF, but there were differences for OSB and PW at the 5\% level. The layer structure and orientation of face fibers in these types of panel could explain these differences.

To understand the interlaminar shear properties of these panels, the shear modulus/strength ratio (GILS/TILS) was obtained. The ratio was around 60 for PW and about 100 for the other panels. This can be explained by the difference in the load-deformation curves shown in Fig. 3. Mat-formed panels appear to be more fragile than PW, so PW has higher shear strength in this test.

Interlaminar shear strength can be evaluated using several methods. The block shear test and Minnesota shear test $^{6,7}$ provide information on strength without having to glue test pieces to steel blocks, and Suchsland proposed a compression shear test as another method. ${ }^{12}$ Shear strength is often compared with IB strength because failures occur in the core layer of panels. Figure 5 shows the relation between TILS and IB strength for the samples, excluding PW. There was a high correlation between TILS and IB for all of the mat-formed panels tested. The regression equation for the line through these points is

$y=1.2+1.4 x \quad R=0.86$

where $y$ and $x$ are TILS and IB strength (MPa), respectively. The interlaminar shear strength and IB strength are
Table 4. Shear properties obtained by the edgewise shear test

\begin{tabular}{|c|c|c|c|c|c|}
\hline \multirow[t]{2}{*}{ Panel type } & \multicolumn{2}{|c|}{ GEWS } & \multicolumn{2}{|c|}{ TEWS } & \multirow[t]{2}{*}{ GEWS/TEWS } \\
\hline & $\mathrm{MPa}$ & CV (\%) & $\mathrm{MPa}$ & CV (\%) & \\
\hline \multicolumn{6}{|l|}{ OSB } \\
\hline $\operatorname{Pr}$ & 1.79 & 22 & 9.48 & 9 & 189 \\
\hline $\mathrm{Pp}$ & 1.71 & 16 & 9.34 & 11 & 183 \\
\hline \multicolumn{6}{|l|}{$\mathrm{PB}$} \\
\hline $\operatorname{Pr}$ & 1.19 & 7 & 8.70 & 11 & 137 \\
\hline $\mathrm{Pp}$ & 1.12 & 10 & 8.89 & 9 & 126 \\
\hline \multicolumn{6}{|l|}{ MDF9 } \\
\hline $\operatorname{Pr}$ & 1.04 & 7 & 12.5 & 5 & 83 \\
\hline $\mathrm{Pp}$ & 1.10 & 12 & 12.5 & 5 & 88 \\
\hline \multicolumn{6}{|l|}{ MDF7 } \\
\hline Pr & 1.12 & 19 & 13.6 & 14 & 82 \\
\hline $\mathrm{Pp}$ & 1.16 & 13 & 14.0 & 11 & 83 \\
\hline \multicolumn{6}{|l|}{ PW } \\
\hline $\operatorname{Pr}$ & 0.65 & 23 & 5.36 & 8 & 122 \\
\hline $\mathrm{Pp}$ & 0.57 & 13 & 5.63 & 10 & 100 \\
\hline
\end{tabular}

GEWS, edgewise shear modulus; TEWS, edgewise shear strength; Pr, parallel to the machine direction or face grain fiber alignment; Pp, perpendicular to the machine direction or the face fiber

closely related and constitute alternative methods for determining IB strength, as reviewed by Kufner. ${ }^{21}$ This also corresponds to the results obtained by Liiri et al., ${ }^{22}$ who determined the strength of the core layer using the shear test and torsion shear test. The results show that IB strength accurately predicts TILS with certain accuracy.

Shear properties by the edgewise shear

The edgewise shear test was used as a third method to examine the bond quality of the panels. Although the ASTM D $1037^{1}$ does not require that the shear modulus be determined, wire-strain gauges were used to detect the strain in the diagonal direction in the test pieces. ${ }^{18}$ The shear modulus and shear strength for the five types of panel in each direction are given in Table 4. There were no significant differences for different directions within the panel for either modulus (GEWS) or strength (TEWS), as is well known in theory. In a simple comparison of the mean values, OSB had the highest GEWS, and MDFs had the highest TEWS in the edgewise shear test; PW was the weakest in both GEWS and TEWS. The edgewise shear modulus/ strength ratio (GEWS/TEWS) is also shown in Table 4. The relation between modulus and strength varied with panel type, even for mat-formed panels, although the interlaminar shear ratios shown in Table 3 were similar. The high shear properties of OSB could be due to the degree of strand alignment. The grain of the strands may not always coincide with the cardinal alignment direction, and these randomly oriented strands could be factors that determine the edgewise shear properties.

\section{Relation between GILS and GEWS}

The interlaminar and edgewise shear tests are both specified in ASTM D 1037, ${ }^{1}$ but these tests are not often con- 


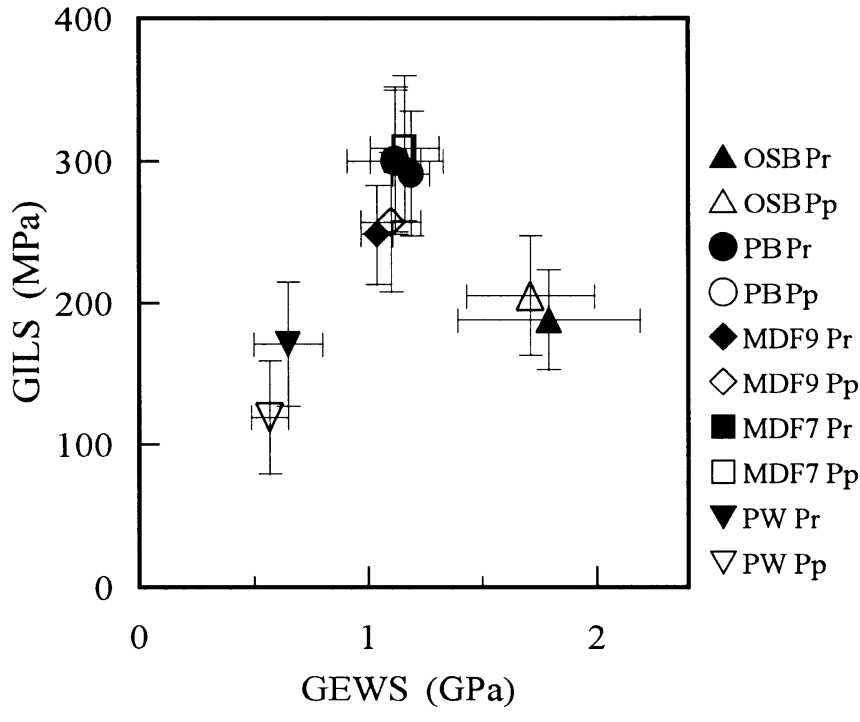

Fig. 6. Relation between the shear modulus obtained by the ILS and the edgewise shear modulus test (GEWS)

ducted because they are difficult to execute. Information on shear modulus is limited for both edgewise and interlaminar shear. To evaluate bond quality, the shear moduli of the wood-based panel products were compared. Figure 6 shows the relation between GILS and GEWS with mean values and standard deviations. Three groups of panels are seen in Fig. 6: OSBs (strand-based panels); PWs (veneer-based panels); and MDF/PBs (fiber- or particle-based panels). The groups were distinctly different, reflecting their characteristic shear properties. MDF/PBs had the highest GILS values, whereas OSBs had the highest GEWS value. One reason for the higher GILS of the MDF/PBs might be that these panels consist of relatively small elements, and not all the elements are laid down in the plane direction of the panels, resulting in out-of-plane orientation angles. A similar logic could be applied to explain the high edgewise shear modulus of OSB. As is well known, there is a range of alignment angles of the strands in each layer of the OSBs; elements with different orientations could strongly resist shear deformation in the plane. Figure 6 reveals that the shear modulus is strongly affected by the panel type.

\section{Conclusions}

Three mechanical tests were conducted to examine the bond quality within wood-based panels made of different elements: internal bond test, interlaminar shear test, and edgewise shear test. The following conclusions were drawn from the results: Epoxy resin, instead of hot-melt glue, is suitable for measuring deformation to determine GILS. There was a linear relation between panel density and GILS, as with the other mechanical properties. This study confirmed the linear correlation between TILS and IB in the mat-formed panels tested. OSB had the highest GEWS, whereas MDFs had the highest strength in the edgewise shear test. The shear modulus and strength depended on the panel type, and the modulus/strength ratio depended on both panel type and loading mode. The relation between GEWS and GILS clearly reflected the characteristics of the shear properties of the panels with different elements.

Acknowledgments We thank Professor Dr. Sobue and Mr. Ikeda, Shizuoka University, for their valuable suggestions throughout the course of this study.

\section{References}

1. American Society for Testing and Materials (1993) Standard test method for properties of wood-based fiber and particle panel materials. ASTM D 1037

2. European Standard (1993) Particleboards and fiberboards: determination of tensile strength perpendicular to the plane of the board. EN319

3. Japanese Industrial Standard (1994) JIS standard specification for particleboard, JIS A-5908

4. Japanese Agricultural Standard (2000) JAS for structural panel

5. European Standard (1993) Timber structures: test methods determination of mechanical properties of wood based panels. prEN-789

6. Hall HJ, Haygreen JG (1983) The Minnesota shear test. Forest Prod J 33:29-32

7. Hall HJ, Haygreen JG, Lee Y (1984) Minnesota shear test reproducibility and correlation with internal bond. Forest Prod J 34:4952

8. McNatt JD (1973) Basic engineering properties of particleboard. In: Proceedings of the 7th Washington State University symposium on particleboard, Pullman, WA, pp 367-385

9. Schulte M, Fruehwald A (1996) Shear modulus, internal bond and density profile of medium density fibre board (MDF). Holz Roh Werkstoff 54:49-55

10. Suzuki S, Piao CX, Saito F (1987) A study evaluating the internal bond properties of MDF (in Japanese). Bull Fac Agr Shizuoka Univ 37:55-60

11. Bateman JH, Hunt MO, Sun CT (1990) New interlaminar shear test for structural wood composites. Forest Prod J 40:9-14

12. Suchsland O (1977) Compression shear test for determination of internal bond strength in particleboard. Forest Prod J 27:32-36

13. Noack D, Schwab E (1972) The shear strength of particleboard as a criterion of particle bonding (in German). Holz Roh Werkstoff 30:440-444

14. Shen KC, Carroll MN (1969) A new method for evaluation of internal strength of particleboard. Forest Prod J 19:17-22

15. Shen KC (1970) Correlation between internal bond and the shear strength measured by twisting thin plates of particleboard. Forest Prod J 20:16-20

16. Gaudert P (1974) New torsion test for particleboard. Forest Prod J 24:35-37

17. Gertjejansen RO, Haygreen JG (1971) Torsion shear test for particleboard adapted to a universal testing machine. Forest Prod J 21:59-60

18. Suzuki S, Nawa D, Miyamoto K, Shibusawa T (2000) Shearthrough-thickness properties of wood-based panels determined by the two-rail shear and edgewise shear methods (in Japanese). J Soc Mater Sci Jpn 49:395-400

19. Lee AWC, Stephens CB (1988) Comparative shear strength of seven types of wood composite panels at high and medium relative humidity conditions. Forest Prod J 38:49-52

20. Suematsu A, Shimoda Y (1998) Evaluation of internal bond quality by interlaminar shear test (in Japanese). In: Abstracts of the 48th annual meeting of the JWRS, p 279

21. Kufner M (1975) Testing the bonding strength of particleboard (in German). Holz Roh Werkstoff 33:265-270

22. Liiri O, Kivistoe A, Tuomimen M, Aho M (1980) Determination of the internal bond of particleboard and fiberboard. Holz Roh Werkstoff 38:185-193 